\title{
HABILIDADE PRAGMÁTICA E SÍNDROME DE DOWN
}

\author{
Pragmatic ability and Down's syndrome
}

\author{
Etelvina Maria França Soares ${ }^{(1)}$, Mônica Medeiros de Britto Pereira ${ }^{(2)}$, Tania Maria Marinho Sampaio ${ }^{(3)}$
}

\begin{abstract}
RESUMO
Objetivo: investigar as habilidades pragmáticas em sujeitos com Síndrome de Down e sua influência no uso comunicativo. Métodos: avaliação da pragmática (ABFW). Participaram desta pesquisa 10 crianças e adolescentes, de ambos os sexos, com idades entre sete e treze anos. A coleta de dados foi realizada a partir de uma situação de brincadeira livre com um familiar. Os padrões de comportamento foram analisados por meio das funções comunicativas, dos meios de comunicação (vocal, gestual ou verbal) e dos atos comunicativos. Os resultados foram tratados estatisticamente $(p<0.05$ Kruskal-Wallis; Q1 = n/4 Q2 = n/2 Q3 =3n/4 $(n=10)$ Primeiro Quartil, Mediana, Terceiro Quartil. Resultados: foram realizados em média 2.88 atos comunicativos por minuto. Entre as funções comunicativas as de maior frequência foram a comentário e a narrativa. O meio comunicativo de maior frequência foi o verbal. Todos os participantes utilizaram as funções comunicativas: comentário, reconhecimento do outro e jogo compartilhado e $90 \%$ do direcionamento da comunicação foi realizado pelas crianças e adolescentes. Conclusão: os meios comunicativos verbal e gestual foram os mais utilizados pelos participantes do estudo. Em relação às funções comunicativas observou-se a predominância da função comentário e narrativa. O direcionamento da comunicação foi realizado predominantemente pelas crianças e adolescentes participantes do estudo.
\end{abstract}

DESCRITORES: Desenvolvimento de Linguagem; Narrativa; Síndrome de Down; Meios de Comunicação

\section{INTRODUÇÃO}

A criança durante os primeiros cinco anos de vida adquire progressivamente as diferentes estruturas da língua materna. Após esta idade, o infante, apreende a sutileza das habilidades da pragmática, utilizando, de forma progressiva, a linguagem compreensiva e a expressiva em contextos específicos. Aos 12 anos de idade o sujeito ainda não domina a variação dos traços de linguagem de acordo com as situações da comunicação ${ }^{1,2}$.

A criança compreende inicialmente as expressões faciais e linguísticas com base no contexto físico que a comunicação acontece. As primeiras

(1) Fonoaudióloga; Professora Titular das Faculdades Pestalozzi, Niterói, RJ; Mestre em Fonoaudiologia pela Universidade Veiga de Almeida.

(2) Fonoaudióloga; Professora Adjunta Universidade Veiga de Almeida, UVA, Rio de Janeiro, RJ; Doutora em Linguística pela Universidade Federal de Minas Gerais.

(3) Graduada em Letras; Professora Adjunta Universidade Veiga de Almeida, UVA, Rio de Janeiro, RJ; Doutora em Filosofia pela Universidade Gama Filho.

Conflito de interesses: inexistente tentativas de comunicação são próximas ao "aqui e agora", geralmente baseadas em situações de rotina com os outros. As crianças, ao interpretarem as expressões linguísticas, confiam muito mais no seu conhecimento de mundo, do que, no significado linguístico intrínseco da própria expressão ou ainda no significado proposto por um interlocutor ${ }^{3,4}$.

A linguagem é uma prática comunicativa. A teoria dos atos da fala é um importante instrumento para interpretar a interação entre os interlocutores. Os atos de fala apresentam uma concepção sistemática para classificar os usos comunicativos da língua. $\mathrm{O}$ ato de fala implica em uma relação intersubjetiva, é um ato comum a falantes e ouvintes e, para tanto, pressupõe ação e reação adequadas de ambas as partes. Alguns autores concordam sobre a importância do estudo da frase como um todo, ressaltando a necessidade da observação da emissão frasal em um contexto global nas ações ${ }^{5,6}$.

A análise pragmática preocupa-se com o contexto, envolvendo a semântica, a sintaxe e a coerência da narrativa. O uso funcional das habilidades da linguagem pode ser percebido através da intersubjetividade das habilidades individuais observada no comportamento onde a comunicação ocorre, 
nos elementos linguísticos e não-linguísticos e no desenvolvimento afetivo e cognitivo ${ }^{7-9}$.

A pragmática envolve três grandes habilidades de comunicação: 1 . uso da linguagem - cumprimento, informação, promessa, pedido. 2. mudança da linguagem - utilizada segundo as necessidades do ouvinte ou da situação, (por ex. linguagem diferente para as babás e para os pais). 3. compreensão das regras para as conversações e narrativas, que considera os turnos de conversação, as expressões faciais e o contato ocular, a interrupção e o recomeço de um tópico de conversação com um interlocutor $^{3-6}$. Assim, a pragmática é constituída por "um conjunto de regras relacionadas com o uso da linguagem em um contexto comunicativo" ${ }^{10}$. Referese à maneira como a linguagem é utilizada para se comunicar, e não à forma como está estruturada. Uma reflexão crítica da pragmática e da análise do discurso pode ser obtida por meio da apreciação do diálogo em um contexto. Ela se justifica quando vamos ao encontro da questão-problema, de uma situação comunicativa, para posteriormente, questionar a validade do discurso ${ }^{11,12}$.

A Síndrome de Down (SD) é uma anormalidade genética comum identificada ao nascimento e pode até mesmo causar desordem intelectiva severa. Ao contrário das expectativas dos profissionais da saúde e dos educadores, o modelo epidemiológico e as mudanças demográficas indicam que nos próximos anos o número de crianças e adultos com SD provavelmente crescerá ao invés de cair ${ }^{13}$.

Estudos relatam que os indivíduos com SD, possuem tempo significativamente menor para a memorização da sentença (memória a curto prazo) ${ }^{14,15}$. Estes dados foram sugeridos com base na análise da neuroimagem do cérebro sem alteração, que retrata a produção vocálica, consonantal e silábica com imensa atividade no cerebelo, assim como, no giro frontal, córtex pré-motor, córtex auditivo e tálamo ${ }^{16}$, áreas estas comprometidas na SD. Na SD observa-se também prejuízo na memória por disfunção do hipocampo, apontando para uma redução múltipla na quantidade da função sináptica em ambos os impulsos, excitatório e inibitório, nos neurônios da via piramidal do hipocampo. No entanto, a potência das conexões sinápticas associativas entre os neurônios piramidais são não apenas abundantes como também normais. A diminuição total do impulso da função sináptica piramidal e a hiperconexão associativa dos neurônios resulta na alteração que pode contribuir para a diminuição da memória observada na SD ${ }^{17}$.

Em relação a outras crianças com atraso no desenvolvimento, a criança com SD apresenta alterações no ato comunicativo e dificuldades para comunicar-se com clareza ${ }^{18}$.
O uso dos gestos comunicativos na SD funciona como um facilitador devido à ininteligibilidade da fala. O desenvolvimento da comunicação gestual na SD segue o da criança com desenvolvimento típico com permanência, no entanto, dos gestos dêiticos por um período de tempo maior ${ }^{19,20}$.

As primeiras palavras geralmente são pronunciadas por volta dos 24 meses. Foi observado que, quando as crianças com SD começam a combinar duas e três palavras dentro da mesma emissão (normalmente por volta dos quatro ou cinco anos de idade), parecem expressar a mesma faixa de significados que a registrada nos estudos de aquisição da linguagem normal ${ }^{21}$.

Objetiva-se assim investigar as habilidades pragmáticas em sujeitos com Síndrome de Down e sua influência no uso comunicativo.

\section{MÉTODOS}

O presente estudo teve caráter quantitativo e qualitativo e dele fizeram parte todos os alunos da Escola Experimental Helena Antipoff, da Associação Pestalozzi de Niterói, portadores de Síndrome de Down, somando-se um total de 10 sujeitos com idades entre sete e 13 anos, sendo três meninas e sete meninos.

Os 10 participantes da pesquisa foram filmados (Canon ES50/ES55) em sessão individual durante 30 minutos em situação de jogo livre, na qual cada sujeito aparece em interação com um familiar que se disponibilizou a participar do estudo.

A seleção dos familiares dos participantes ficou a critério da disponibilidade destes em função do seu trabalho ou dos afazeres domésticos. Compareceram então, nove mães e uma irmã.

O material utilizado para a situação de jogo livre constou de carrinhos, blocos de encaixe, bonecos, bolas de vários tamanhos (vôlei, futebol e Bobath), lápis de cor, hidrocor, papel, massa de modelar, miniaturas. Também foram utilizados jogos variados em perfeito estado e brinquedos quebrados, estes últimos, com a finalidade de analisar a reação dos sujeitos frente aos objetos imperfeitos no processo comunicativo.

Durante as filmagens a pesquisadora permaneceu no mesmo local da sala, situando a dupla filmada no perímetro de um metro quando esta se encontrava nos tatames com os brinquedos de montagem, ou sentada na mesa com material de desenho, ou ainda, a uma distância de até quatro metros quando o sujeito avaliado ou a dupla (criança/familiar) jogava bola ou se deslocava pela sala.

Utilizou-se como parâmetro de análise da linguagem pragmática nos sujeitos com Síndrome de 
Down a Avaliação da Pragmática de Fernandes ${ }^{22}$. A proposta da avaliação é facilitar a decisão diagnóstica através de parâmetros quantitativos e análise qualitativa ampliando a compreensão da comunicação humana.

Esta pesquisa foi aprovada pelo Comitê de Ética em Pesquisa da Universidade Veiga de Almeida (Protocolo número 04/04).

Neste trabalho, foram utilizados os testes não paramétricos de Kruskal-Wallis $(p<0.05){ }^{23}$ e as medidas descritivas: $\mathrm{Q} 1=\mathrm{n} / 4$ Primeiro Quartil, $\mathrm{Q} 2=$ n/2 Mediana, $Q 3=3 n / 4$ Terceiro Quartil. $(n=10)^{24}$.

\section{RESULTADOS}

Os resultados obtidos na análise do vídeo definiram os dados do Perfil Comunicativo e se referem: ao número de atos comunicativos por minuto (interação entre os interlocutores ou entre estes e o objeto, desde o inicio da ação até o término desta), ao percentual de atos comunicativos expressos pela criança (espaço de comunicação que o sujeito possui para se expressar), aos meios comunicativos utilizados (verbal, gestual, vocal), às funções comunicativas (ação utilizada pelo sujeito para se comunicar) mais frequentes, ao número de funções comunicativas expressas e ao direcionamento da comunicação (registro de quem inicia a atividade, participante ou adulto).

O valor da mediana dos atos comunicativos por minuto entre os 10 participantes foi de 2.88
Tabela 1 - Medidas descritivas dos atos comunicativos por minuto

\begin{tabular}{cccc}
\hline & $\begin{array}{c}\text { Primeiro } \\
\text { Quartil }\end{array}$ & Mediana & $\begin{array}{c}\text { Terceiro } \\
\text { Quartil }\end{array}$ \\
\hline $\begin{array}{c}\text { Atos } \\
\text { comum. } \\
\text { p/ min. }\end{array}$ & 2,23 & 2,88 & 3,48 \\
\hline Nota: $\mathrm{Q} 1=\mathrm{n} / 4$ & $\mathrm{Q} 2=\mathrm{n} / 2$ & $\mathrm{Q} 3=3 \mathrm{n} / 4 \quad(\mathrm{n}=10)$
\end{tabular}

(Tabela 1). A seguir encontra-se a descrição do percentual apurado das funções comunicativas utilizadas pelo número total $(n=10)$ dos participantes (Figura 1):

- as funções: Comentário, Reconhecimento do Outro e Jogo Compartilhado, foram utilizadas por 10 participantes (100\%);

- as funções: Exploratória, Jogo, Pedido de Ação e Exclamativo, ocorreram em nove participantes (90\%) dos 10 sujeitos avaliados;

- a função Pedido de Informação foi utilizada por oito sujeitos $(80 \%)$ na interação entre os 10 interlocutores;

- a Nomeação atingiu sete crianças/adolescentes (70\%) dos 10 sujeitos avaliados;

- a função Narrativa ocorreu em seis sujeitos $(60 \%)$ entre os 10 participantes;

- Performativo e Exibição ocorreram em cinco sujeitos $(50 \%)$ na comunicação entre as 10 crianças e ou adolescentes avaliadas;

\begin{tabular}{|c|c|c|c|c|c|c|c|c|c|c|}
\hline \multicolumn{11}{|c|}{ PERFIL COMUNICATIVO } \\
\hline Participantes & 1 & 2 & 3 & 4 & 5 & 6 & 7 & 8 & 9 & 10 \\
\hline $\begin{array}{l}\text { Atos comun/ } \\
\text { por min. }\end{array}$ & 1.73 & 2.70 & 2.73 & 2.06 & 2.06 & 3.13 & 3.03 & 4.07 & 3.60 & 4.03 \\
\hline $\begin{array}{c}\% \text { atos } \\
\text { comunicativos }\end{array}$ & 78.46 & 82.65 & 78.09 & 79.48 & 68.88 & 64.00 & 81.98 & 81.87 & 74.48 & 85.81 \\
\hline \multirow{3}{*}{$\begin{array}{c}\text { Meios } \\
\text { comunicativos }\end{array}$} & Vo 62; & 20 & 12 & -- & 3 & 41 & 15; & -- & -- & -- \\
\hline & G 37; & 29 & 21 & 29 & 80 & 40 & 49 & 14 & 48 & 55 \\
\hline & Ve -- & 49 & 65 & 70 & 16 & 17 & 35 & 85 & 52 & 45 \\
\hline \multirow{2}{*}{ Direcionamento. } & A $22 \%$ & $17 \%$ & $78 \%$ & $21 \%$ & $31 \%$ & $36 \%$ & $18 \%$ & $21 \%$ & $26 \%$ & $14 \%$ \\
\hline & C $78 \%$ & $83 \%$ & $22 \%$ & $79 \%$ & $69 \%$ & $64 \%$ & $82 \%$ & $79 \%$ & $74 \%$ & $86 \%$ \\
\hline $\begin{array}{l}\text { № funções } \\
\text { comunicativas }\end{array}$ & 9 & 11 & 10 & 11 & 13 & 12 & 11 & 14 & 12 & 10 \\
\hline $\begin{array}{c}\text { Frequência } \\
\text { das funções } \\
\text { comunicativas }\end{array}$ & $\begin{array}{lr}\text { C } & 39 \\
\text { PR } & 15 ; \\
\text { J } & 11 ; \\
\text { XP } & 7 ; \\
\text { EX } & 7 ;\end{array}$ & $\begin{array}{lr}\text { C } & 23 ; \\
\text { RO } & 14 ; \\
J & 14 ; \\
\text { XP } & 13 ; \\
\text { N } & 11 ;\end{array}$ & $\begin{array}{l}\text { EX } 28 ; \\
\text { JC } 15 ; \\
\text { N } 14 ; \\
\text { C } 14 ; \\
\text { NA } 7 ;\end{array}$ & $\begin{array}{l}\text { JC } 25 ; \\
\text { C 20; } \\
\text { N 17; } \\
\text { NA14; } \\
\text { RO } 6 ;\end{array}$ & $\begin{array}{l}\text { EX 33; } \\
\text { XP 16; } \\
\text { C 11; } \\
\text { JC 8; } \\
\text { E 8; }\end{array}$ & $\begin{array}{lr}\text { C } & 27 ; \\
\text { XP } & 17 ; \\
\text { PI } & 13 ; \\
\text { JC } & 12 ; \\
\text { RO } & 8 ;\end{array}$ & $\begin{array}{lr}\text { C } & 29 ; \\
\text { JC } & 15 ; \\
\text { XP } & 9 ; \\
\text { PA } & 9 ; \\
\text { PI } & 8 ;\end{array}$ & $\begin{array}{ll}\text { C } & 39 \\
\text { NA } & 15 ; \\
\text { PI } & 9 ; \\
\text { RO } & 8 ; \\
\text { JC } & 4 ;\end{array}$ & $\begin{array}{lr}\text { RO } & 16 ; \\
\text { JC } & 14 ; \\
\text { C } & 12 ; \\
\text { PR } & 11 ; \\
\text { EX } & 8 ;\end{array}$ & $\begin{array}{lr}\text { RO } & 24 ; \\
\text { EX } & 21 ; \\
\text { C } & 6 ; \\
\text { PI } & 12 ; \\
\text { JC } & 7 ;\end{array}$ \\
\hline
\end{tabular}

Legenda: Meios Comunicativos: VO - Vocal G - Gestual VE - Verbal.

Direcionamento: A - Adulto C - Criança.

Funções Comunicativas: C - Comentário; EX - Exclamativo; JC - Jogo Compartilhado; RO - Reconhecimento do Outro; PR - Protesto; XP - Exploratória; NA - Narrativa; J - Jogo; N - Nomeação; PI - Pedido de Informação; P O - Pedido de Objeto; PA Pedido de Ação; E - Exibição; PE - Performativo; RE - Reativos; EP - Expressão de Protesto. 
- a função Protesto obteve como resposta predominante em quatro participantes (40\%) dos 10 sujeitos avaliados;

- as funções Expressão de Protesto e Reativos obtiveram predominância em três sujeitos (30\%) na comunicação entre os 10 interlocutores;

- Pedido de Rotina Social ocorreu em dois (20\%) entre os 10 sujeitos avaliados;

- as funções Não Focalizada, e Pedido de Objeto ocorreram em um sujeito (10\%) entre os 10 interlocutores.

Os participantes (90\%) foram responsáveis pelo predomínio das ações de direcionamento da comunicação em comparação com apenas um dos familiares (10\%) (Figura 1).

A análise do uso das funções comunicativas por participante apontou a função comentário como a mais predominante nos dez participantes, sendo identificada em (50\%) das interações. As funções: exclamativo e reconhecimento do outro aparecem em segundo lugar totalizando cada uma (20\%) entre os dez sujeitos. E ainda, como terceira opção mais frequente, destaca-se a função jogo compartilhado, reunindo $10 \%$ do total de participantes (Figura 1).

Em relação aos meios comunicativos, constatou-se que cinco sujeitos (50\%) apresentaram predominância do uso do meio verbal, três sujeitos $(30 \%)$ do gestual, e dois sujeitos $(20 \%)$ do meio vocal (Figura 1).

O meio comunicativo verbal destaca neste grupo como o de maior significância (Tabela 2). A análise realizada com as funções comunicativas demonstrou que a função comentário ocorreu significativamente mais do que função jogo e do que a função Performativo. Já a função narrativa ocorreu mais do que a Comentário (Tabela 3).

A Figura 2 apresenta o percentual de utilização dos meios comunicativos e das funções comunicativas de cada participante, como descrito a seguir:

Sujeito 1 - predomínio no meio Vocal das funções Protesto e Performativo em (100\%) e da função Pedido de Ação em (50\%).

Sujeito 2 - predomínio no meio Vocal da função Jogo em (66\%) e a função Exibição em (100\%). No meio Verbal houve o predomínio da função Jogo em $(42 \%)$.

Sujeito 3 - predomínio no meio Vocal da função Exclamativo em (71\%). No meio Gestual, a função Performativo destacou-se em (60\%) e, no meio Verbal, as funções Nomeação em (25\%), Exclamativo em (34\%) e Performativo em (60\%).

Sujeito 4 - predomínio no meio Verbal da função Jogo Compartilhado em (27\%).
Tabela 2 - Valores de significância dos meios comunicativos

\begin{tabular}{ccc}
\hline Parameter & P value & Summary \\
\hline VE vs VO & $p<0.05$ & ${ }^{*}$ \\
\hline VO vs G & $p<0.05$ & ${ }^{*}$ \\
\hline
\end{tabular}

Legenda: $\mathrm{VE}=$ meio comunicativo verbal. $\mathrm{VO}=$ meio comunicativo vocal. $\mathrm{G}=$ meio comunicativo gestual

Tabela 3 - Valores de significância das funções comunicativas

\begin{tabular}{ccc}
\hline Parameter & P value & Summary \\
\hline C vs J & $p<0.05$ & ${ }^{*}$ \\
\hline C vs PE & $p<0.001$ & ${ }^{*}$ \\
\hline NA vs C & $p<0.01$ & ${ }^{*}$ \\
\hline
\end{tabular}

Legenda: $\mathrm{C}=$ função comunicativa comentário. $\mathrm{J}=$ função comunicativa jogo. PE = função comunicativa performativo. $\mathrm{NA}$ = função comunicativa narrativa

Sujeito 5 - predomínio no meio Gestual das funções Exploratória em (21\%), Exibição em (38\%), Pedido de Ação em (22\%), assim como, as funções Expressão de Protesto, Pedido de Consentimento, Pedido de Rotina Social, Não Focalizada destacaram-se em (100\%).

Sujeito 6 - predomínio no meio Vocal das funções Comentário em (78\%), Exploratória em (75\%), Pedido de Ação em (50\%) e Pedido de Rotina Social em (100\%). No meio Gestual a função Jogo Compartilhado em (21\%) e Pedido de Informação em (50\%).

Sujeito 7 - predomínio no meio Vocal das funções Jogo Compartilhado em (62\%) e Reativos em (100\%). No meio Gestual, a função Reativos destacou-se em (57\%). No meio Verbal, surgiram as funções Exploratória em (60\%), Jogo em (42\%), Exibição em (44\%) e Pedido de Ação em (56\%).

Sujeito 8 - predomínio no meio Gestual da função Exibição em (38\%). No meio Verbal, apareceram às funções Comentário em (39\%), Pedido de Informação em (25\%), Narrativa em (51\%), Reconhecimento do Outro em (34\%), Expressão de Protesto em (50\%), Pedido de Objeto em (100\%) e Reativos em (100\%).

Sujeito 9 - predomínio no meio Gestual das funções Protesto em (40\%) e Pedido de Ação em (22\%). No meio Verbal, a função Exibição destacou-se em $(44 \%)$ dos casos.

Sujeito 10 - predomínio no meio Gestual das funções Comentário em (55\%), Reconhecimento do Outro em (30\%), Exclamativo em (34\%), Jogo em (29\%), Pedido de Informação em (50\%), Pedido de Rotina de Ação em (22\%). No meio Verbal, a função Protesto apareceu em (100\%) das interações. 


\begin{tabular}{|c|c|c|c|}
\hline $\begin{array}{c}\text { Meio } \\
\text { comunicativo }\end{array}$ & $\begin{array}{c}\text { Função } \\
\text { comunicativa }\end{array}$ & $\%$ & $\begin{array}{l}\text { Valor referente (\%) dos participantes nas funções comunicativas com } \\
\text { relação aos meios comunicativos }\end{array}$ \\
\hline \multirow{11}{*}{ VOCAL } & $\mathrm{C}$ & 32.35 & Sujeito 6 utilizou $78 \%$ da função C no meio vocal. \\
\hline & $\mathrm{XP}$ & 15.68 & Sujeito 6 utilizou $75 \%$ da função XP no meio vocal. \\
\hline & $\mathrm{JC}$ & 15.68 & Sujeito7 utilizou $62 \%$ da função JC no meio vocal. \\
\hline & EX & 13.72 & Sujeito 3utilizou $71 \%$ da função EX no meio vocal. \\
\hline & $\mathrm{J}$ & 8.82 & Sujeito 2 utilizou $66 \%$ da função $\mathrm{J}$ no meio vocal. \\
\hline & PR & 3.92 & Sujeito 1utilizou $100 \%$ da função PR no meio vocal. \\
\hline & RE & 3.92 & Sujeito 7 utilizou $100 \%$ da função RE no meio vocal. \\
\hline & PA & 1.96 & Sujeitos 1 e 6 utilizaram $50 \%$ cada um da função PA no meio vocal. \\
\hline & $\mathrm{PE}$ & 1.96 & Sujeito 1 utilizou 100\% da função PE no meio vocal. \\
\hline & E & 0.98 & Sujeito 2 utilizou $100 \%$ da função $E$ no meio vocal. \\
\hline & PS & 0.98 & Sujeito 6 utilizou $100 \%$ da função PS no meio vocal. \\
\hline \multirow{16}{*}{ GESTUAL } & $\mathrm{C}$ & 24.39 & Sujeito 10 utilizou $55 \%$ da função C no meio gestual. \\
\hline & RO & 17.31 & Sujeito 10 utilizou $30 \%$ da função RO no meio gestual. \\
\hline & $\mathrm{JC}$ & 13.65 & Sujeito 6 utilizou $21 \%$ da função JC no meio gestual. \\
\hline & $\mathrm{XP}$ & 11.21 & Sujeito 5 utilizou $21 \%$ da função XP no meio gestual. \\
\hline & EX & 10.48 & Sujeito 10 utilizou 34\% da função EX no meio gestual. \\
\hline & $\mathrm{J}$ & 6.58 & Sujeito 10 utilizou $29 \%$ da função $\mathrm{J}$ no meio gestual. \\
\hline & PR & 3.65 & Sujeito 9 utilizou $40 \%$ da função PR no meio gestual. \\
\hline & E & 3.17 & Sujeitos 5 e 8 utilizaram $38 \%$ cada um da função E no meio gestual. \\
\hline & $\mathrm{PI}$ & 2.92 & Sujeitos 6 e 10 utilizaram 50\% cada um da função PI no meio gestual. \\
\hline & $\mathrm{PA}$ & 2.19 & Sujeitos 5, 9 e 10 utilizaram $22 \%$ cada um da função PA no meio gestual. \\
\hline & $\mathrm{RE}$ & 1.70 & Sujeito 7 utilizou $57 \%$ da função RE no meio gestual. \\
\hline & $\mathrm{PE}$ & 1.21 & Sujeito 3 utilizou $60 \%$ da função PE no meio gestual. \\
\hline & EP & 0.73 & Sujeito 5 utilizou 100\% da função EP no meio gestual. \\
\hline & PC & 0.24 & Sujeito 5 utilizou $100 \%$ da função PC no meio gestual. \\
\hline & PS & 0.24 & Sujeito 5 utilizou $100 \%$ da função OS no meio gestual. \\
\hline & NF & 0.24 & Sujeito 5 utilizou $100 \%$ da função NF no meio gestual. \\
\hline \multirow{16}{*}{ VERBAL } & $\mathrm{C}$ & 29.80 & Sujeito 8 utilizou $39 \%$ da função $C$ no meio verbal. \\
\hline & $\mathrm{N}$ & 11.57 & Sujeito 3 utilizou $25 \%$ da função $\mathrm{N}$ no meio verbal. \\
\hline & $\mathrm{PI}$ & 11.57 & Sujeito 8 utilizou $25 \%$ da função PI no meio verbal. \\
\hline & EX & 9.35 & Sujeito 3 utilizou 34\% da função EX no meio verbal. \\
\hline & NA & 9.11 & Sujeito 8 utilizou 51\% da função NA no meio verbal. \\
\hline & $\mathrm{JC}$ & 7.14 & Sujeito 4 utilizou $27 \%$ da função JC no meio verbal. \\
\hline & RO & 5.66 & Sujeito 8 utilizou $34 \%$ da função RO no meio verbal. \\
\hline & PA & 3.94 & Sujeito 7 utilizou $56 \%$ da função PA no meio verbal. \\
\hline & E & 2.21 & Sujeitos 7 e 9 utilizaram $44 \%$ cada um da função $E$ no meio verbal. \\
\hline & EP & 1.97 & Sujeito 8 utilizou $50 \%$ da função EP no meio verbal. \\
\hline & $\mathrm{J}$ & 1.72 & Sujeitos 2 e 7 utilizaram $42 \%$ cada um da função $\mathrm{J}$ no meio verbal. \\
\hline & $\mathrm{PE}$ & 1.23 & Sujeito 3 utilizou $60 \%$ da função PE no meio verbal. \\
\hline & $\mathrm{XP}$ & 1.23 & Sujeito 7 utilizou $60 \%$ da função XP no meio verbal. \\
\hline & $\mathrm{PO}$ & 0.73 & Sujeito 8 utilizou $100 \%$ da função PO no meio verbal. \\
\hline & $\mathrm{RE}$ & 0.73 & Sujeito 8 utilizou $100 \%$ da função RE no meio verbal. \\
\hline & PR & 0.49 & Sujeito 10 utilizou $100 \%$ da função PR no meio verbal. \\
\hline
\end{tabular}

Legenda: A porcentagem é o resultado do número total dos atos comunicativos expressos em cada função comunicativa, multiplicado por 100 e dividido pelo número total de atos comunicativos e de todas as funções comunicativas.

Funções Comunicativas: C - Comentário; EX - Exclamativo; JC - Jogo; Compartilhado; RO - Reconhecimento do Outro; PR - Protesto; XP - Exploratória; NA - Narrativa; J - Jogo; N - Nomeação; PI - Pedido de Informação; PO - Pedido de Objeto; PA - Pedido de Ação; E - Exibição; PE - Performativo; RE - Reativos; EP - Expressão de Protesto; PC - Pedido de Consentimento; NF - Não-focalizada; PS - Pedido de Rotina Social.

Figura 2 - Distribuição do valor total dos atos comunicativos expressos no meio vocal gestual e verbal das funções comunicativas e o valor dos participantes nestas funções 


\section{DISCUSSÃO}

O estudo das variabilidades individuais de cada sujeito nas ações comunicativas possibilitou identificar a melhor situação de comunicação no contexto de interação adulto-criança com SD.

A análise individual dos resultados permitiu investigar a melhor abordagem clínica para as questões apresentadas segundo cada caso ${ }^{25}$. A presença de desequilíbrios entre espaço interativo, meio e função comunicativa pode indicar a necessidade de estímulo individual buscando maior equilíbrio. Por exemplo, o sujeito 4 utilizou bastante a função NA e do meio verbal em relação grupo pesquisado, no entanto realizou poucos atos comunicativos por minuto, o que demonstra pouca utilização do espaço interativo.

O desempenho das habilidades da linguagem pode ser observado por meio dos dados da pesquisa que indicam as correlações inapropriadas entre a interatividade da comunicação e o uso do meio de comunicação ${ }^{19,22}$.

O meio comunicativo gestual foi utilizado pelos 10 sujeitos avaliados, enquanto o meio vocal foi utilizado apenas por seis sujeitos $(1,2,3,5,6,7)$ e o meio verbal por nove sujeitos $(2,3,4,5,6,7,8,9,10)$. Esses dados sugerem a utilização dos gestos comunicativos para compensar o atraso na produção oral, buscando se fazer entender melhor ${ }^{19,20}$.

Das 20 funções comunicativas, três delas predominaram em todo o grupo (Comentário, Jogo Compartilhado e Reconhecimento do Outro). A função Comentário neste grupo evidenciou-se por meio de apontar objetos e de nomeações básicas sobre os objetos conhecidos e atividades de vida diária. A utilização desta função na SD é significativa, uma vez que o relato sobre objetos e fatos vividos é realizado nesta clientela em sentenças simples. Os dados reforçam a idéia de que o sujeito com SD possui limitações cognitivas, reduzida capacidade para generalizar e memória limitada para os acontecimentos recentes ${ }^{14-21}$.

A função Reconhecimento do Outro, observada em seis dos participantes, retrata de forma dinâmica a busca de interação neste contexto de avaliação. Esta função caracteriza-se pela obtenção de atenção e demonstra a importância da interação mãecriança como fonte de estímulos cognitivo e linguístico no desenvolvimento, possibilitada através da mediação entre as ações da criança e o meio ${ }^{20,26,27}$.

A função jogo compartilhado, observada em oito participantes, envolve de forma natural e gradativa a interação entre os interlocutores conhecidos ou não, permitindo a esses sujeitos o encontro na divisão com outros indivíduos em propostas lúdicas com grande vivacidade e dinamismo nas atividades. E mais, esta função suscita a possibilidade da construção e do desenvolvimento da representação simbólica por meio da interação ${ }^{28}$.

Os estudos destacam maior predominância da mãe no direcionamento da atividade em jogos e brincadeiras livres com crianças com SD em seus lares ${ }^{21}$. No presente estudo observou-se um panorama diferente, no qual $90 \%$ das crianças foram responsáveis por este direcionamento. Essa falta de direcionamento por parte das mães levou a uma quebra no espaço comunicativo com quatro sujeitos ( $40 \%$ da população avaliada), provocando dispersão, agitação e desinteresse pela proposta em questão.

A interferência familiar não apropriada pode alterar a resposta de um sujeito com prejuízo de linguagem ocasionando sentenças mal organizadas 29. Irmãos de indivíduos com SD, muitas vezes, não executam conversas longas em sua convivência, no entanto, se uma criança aborda um tópico, os pais podem ajudá-la periodicamente perguntando sobre algumas questões, desde que, a informação possa evitar uma confusão desnecessária ${ }^{30}$. Devido a estes fatores salientamos o espaço comunicativo como importante fator e de grande relevância para o estudo do desenvolvimento da linguagem na SD.

A função NA também foi utilizada de forma significativa por alguns participantes através do relato de histórias bem organizadas e contadas a partir do material apresentado na avaliação. A narrativa oral ganha destaque nesta população porque a compreensão do vocabulário é o melhor predicado da habilidade para o grupo com SD ${ }^{31}$.

\section{CONCLUSÃO}

Foram realizados em média 2.88 atos comunicativos por minuto. As crianças e adolescentes participantes do estudo foram responsáveis por $90 \%$ do direcionamento da comunicação. A análise geral do uso dos meios comunicativos apontou o meio verbal e gestual como os mais utilizados. A análise da utilização dos meios por informantes aponta a predominância do meio verbal em $50 \%$ deles, do meio gestual em $30 \%$ do meio vocal em apenas $20 \%$. A análise do uso das funções comunicativas por participante apontou as funções comentário e narrativa como as mais predominantes nos 10 participantes. 


\begin{abstract}
Purpose: to investigate the pragmatic abilities and their influence on the communicative use in subjects with Down's syndrome. Methods: the pragmatic assessment (ABFW). The participants were 10 children and adolescents, male and female, from seven to 13-year old. The behavior patterns were analyzed by using the communicative functions, the communication means (vocal, gestural or verbal) and the communicative acts. Data collection was provided from a free play situation with a family member. The Kruskal Wallis test was used for statistic analysis $(p<0.05 Q 1=n / 4$ Q2 =n/2 Q3 $=3 n / 4(n=10)$ First Quartile, Median, Third Quartile). Results: the median of the communicative acts was 2.88. The comment and narrative functions were the more frequent among the communicative functions and the verbal was the more frequent communicative mean. All the participants used the functions: comment, recognitions of the other and game shared functions. The children and adolescents were responsible for $90 \%$ of the direction of the communication. Conclusion: the verbal and gestural communication means and the comment and narrative functions were the most used among the participants; mothers were responsible for just $10 \%$ of the communication direction and it was carried out predominantly by the children and adolescents that took part in the study.
\end{abstract}

KEYWORDS: Language Development; Narrative; Down's Syndrome; Communications Media

\section{REFERÊNCIAS}

1. Virginie L, Stéphanie C. Comment étudier les capacities pragmatiques des enfants en situation de compréhension du langage? J Title Université de Neuchâtel. 2005; 42:65-79.

2. Kasper G. Four perspectives on L2 pragmatic development. Appl Linguist. 2001; 22(4):502-30.

3. Loukusa S. The use of context in pragmatic language comprehension in normally developing children and children with Asperger syndrome / high-functioning autism. [dissertação] Oulu (Finland): Faculty of Humanities of the University of Oulu; 2007.

4. Loukusa S, Leinonen E, Ryder N. Development of pragmatic language comprehension in Finnishspeaking children. First Lang. 2007; 27(3):279-96.

5. Peccei JS. Pragmatics. London and New York: Routledge Taylor \& Francis Group; 2004.

6. Hage SRV, Resegue MM, Viveiros DCS, Pacheco EF. Análise do perfil das habilidades pragmáticas em crianças pequenas normais. Pró-Fono. 2007 Jan-Abr; 19(1):49-58.

7. Xu J, Kemeny S, Park G, Frattali C, Braun A. Language in context: emergent features of word, sentence, and narrative comprehension. Neuroimage. 2004; 25(3):1002-15.

8. Befi-Lopes DM, Bento ACP, Perissinoto J. Narração de histórias por crianças com distúrbio específico de linguagem. Pró-Fono. 2008; 20(2):93-8.

9. Porto E, Limongi SCO, Santos IG, Fernandes FDM. Amostra de filmagem e análise da pragmática na síndrome de Down. Pró-Fono. 2007; 19(2):159-66.

10. Owens REJ. Desarrollo del lenguaje. Madrid: Pearson Prentice Hall; 2003.

11. Cutting J. Pragmatics and discourse. A resource book for students. London and New York: Routledge Taylor \& Francis Group; 2003.

12. Mallia $P$, Have $H$. Pragmatic approaches to genetic screening. Med Health Care Philos. 2005; 8(1):69-77.

13. Wishart JG. Socio-cognitive understanding: a strength or weakness in Down's syndrome? J Intellect Disab Res. 2007; 51(12):996-1005.

14. Seung HK, Chapman RS. Sentence memory of individuals with Down's syndrome and typically developing children. J Intellect Disab Res. 2004; 48(2):160-71.

15. Chapman RS, Sindberg H, Bridge C, Gigstead K, Hesketh L. Effect of memory support and elicited production on fast mapping of new words by adolescents with Down syndrome. J Speech Hear Res. 2006; 49(1):3-15.

16. Ghosh SS, Tourville JA, Guenther FH. A neuroimaging study of premotor lateralization and cerebellar involvement in the production of phonemes and syllables. J Speech Lang Hear Res. 2008; 51(5):1183-202.

17. Hanson JE, Blank M, Valenzuela RA, Garner CC, Madison DV. The functional nature of synaptic circuitry is altered in area CA3 of hippocampus in a mouse model of Down's syndrome. J Physiol. 2007; 579(1):53-67.

18. Roberts JE, Price J, Malkin C. Language and communication development in Down syndrome. 
Mental Retard Develop Disab Res Rev. 2007; 13(1):26-35.

19. Andrade RV, Limonge SCO. A emergência da comunicação expressiva na criança com síndrome de Down. Pró-Fono. 2007; 19(4):387-92.

20. Porto-Cunha E, Limongi SCO. Modo comunicativo utilizado por crianças com síndrome de Down. Pró-Fono. 2008; 20(4):243-8.

21. Bishop D, Mogford K. Desenvolvimento da linguagem em circunstâncias excepcionais. Rio de Janeiro: Revinter; 2002.

22. Fernandes FDM. Pragmática. In: Andrade CRF, Befi-Lopes DM, Fernandes FDM, Wertzner HF. ABFW: Teste de linguagem nas áreas de fonologia, vocabulário, fluência e pragmática. Carapicuíba (SP): Pró-Fono; 2004. p. 83-7.

23. Siegel S. Estatística não-paramétrica para ciências do comportamento. São Paulo: McGrawHill do Brasil; 1981.

24. Spiegel MR. Estatística. 3. ed. São Paulo: Makron Books; 2004.

25. Fernandes FDM, Miilher LP. Relações entre a Autistic Behavior Checklist (ABC) e o perfil funcional da comunicação no espectro autístico. Pró-Fono. 2008; 20(2):111-6.
26. Spaulding TG, Plante E, Vance R. Sustained selective attention skills of preschool children with specific language impairment: evidence for separate attentional capacities. J Speech Lang Hear Res. 2008; 51(1):16-34.

27. Visootsak J, Sherman S. Neuropsychiatric and behavioral aspects of trisomy 21. Curr Psychiatr Rep. 2007; 9(2):135-40.

28. Alt M, Plante E. Factors that influence lexical and semantic fast mapping of young children with specific language impairment. J Speech Lang Hear Res. 2006; 49(5):941-54.

29. Ford JA, Milosky LA. Inference generation during discourse and its relation to social competence: an online investigation of abilities of children with and without language impairment. J Speech Lang Hear Res. 2008; 51(2):367-80.

30. Skotko BG, Levine SP. What the other children are thinking: brothers and sisters of persons with Down syndrome. Am J Med Genet C Semin Med Genet. 2006; 142C(3):180-6.

31. Kay-Raining Bird E, Cleave PL, White D, Pike H, Helmkay A. Written and oral narratives of children and adolescents with Down syndrome. J Speech Lang Hear Res. 2008; 51(2):436-50.

RECEBIDO EM: 18/08/2008

ACEITO EM: 08/07/2009

Endereço para correspondência:

Etelvina Maria França Soares

Av. Almirante Ari Parreiras, 689 ap. 405

Niterói - RJ

CEP: 24230321

E-mail: etel@ajato.com.br 